\title{
Novel Antiresonant Hollow Core Fiber Design with Ultralow Leakage Loss Using Transverse Power Flow Analysis
}

\author{
Gregory T. Jasion, David J. Richardson, Francesco Poletti \\ Optoelectronics Research Centre, Faculty of Engineering and Physical Sciences, University of Southampton, UK \\ g.jasion@soton.ac.uk
}

\begin{abstract}
By analyzing transverse power flows in hollow core Nested Antiresonant Nodeless Fibers (NANFs), we devise a simple and realistically achievable structural improvement that promises over two orders of magnitude lower leakage loss than current state-of-the-art.

OCIS codes: Fiber design and fabrication (060.2280), Microstructured fibers (060.4005), Fiber optics and optical (060.0060) communications, Fiber properties (060.2400)
\end{abstract}

\section{Introduction}

Hollow core optical fibers guide light in a hollow core surrounded by a microstructured cladding. Replacing the glass core with gas or vacuum promises many advantages to the fields of power delivery, sensing and telecoms, such as higher damage thresholds, reduced non-linearity, latency and environmental sensitivity, as well as the potential for loss as low as, if not superior to, standard single mode fibers (SSMF) [1].

The lowest reported loss in a data-transmitting hollow core fiber is $1.3 \mathrm{~dB} / \mathrm{km}$, recently measured in a Nested Antiresonant Nodeless Fiber (NANF) [2]. To reduce the still considerable loss gap to SSMFs and make hollow core fiber technology even more appealing to optical communications, fiber designers need to find structural ways to reduce the NANF's leakage loss, simulations indicate this is their dominant loss mechanism [2]. Enlarging the core diameter from the current 30um or nesting a second nested ring are two possible routes to achieve that purpose [1]. However, as a downside they make the fiber more susceptible to bend loss and considerably more complicated to fabricate, respectively. Here we use power flow streamlines to study which structural features are associated with the greatest power leakage in NANFs, and exploit the surprising results from the study to develop a novel structure with considerably lower leakage than state-of-the-art fibers. A numerical assessment indicates that improvements of nearly 500 times over the leakage loss of current world-record loss NANFs should be possible, in a fiber with only a modest increase in fabrication complexity.

\section{Visualizing the transverse flow of power}

A NANF is an anti-resonant tubular fiber, where nested tubes of the same thickness are bonded to the inside of a jacket tube, allowing for nodeless arcs of glass surrounding the core, Fig. 1a. This creates multiple radial reflections and avoids junctions or nodes that would add resonances, increase the loss and narrow the bandwidth. Here, we use transverse power flow streamlines to understand how the power guided in a NANF leaves the core and the routes it takes, and use this analysis to generate a fiber design with reduced leakage loss. This visualization technique is familiar to the fluid dynamics community, but has only recently been used to understand hollow core fibers [3]. As we will show, understanding the routes by which power escapes from the core can provide insight into the function of each geometrical element in the cladding geometry. This analysis can then directly inform the design of improved fibers.
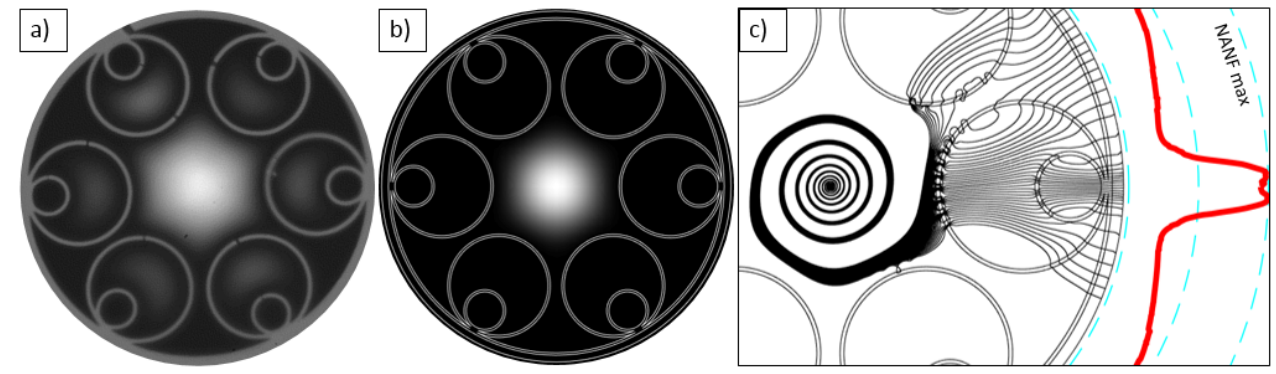

Figure 1 - a) Fabricated NANF fiber and experimental mode-field, credit: Seyed Reza Sandoghchi [2], b) Simulated NANF geometry and the longitudinal Poynting vector, $S_{z}$, of the fundamental core mode, c) Simulated NANF geometry with streamlines following the transverse Poynting vector field. Streamlines are seeded near the center of the core and spiral outwards, illustrating $1 / 6^{\text {th }}$ of the vector field. The red curve shows the density of power leakage at the outer boundary of the geometry, leakage is highest at the center of the tubular elements.

Streamlines are lines that are always parallel to the local vector field, starting positions are specified to seed the streamline which is then advanced by integrating using the local vector field. In a $2 \mathrm{D}$ vector field with orthogonal components $u(x, y)$ and $v(x, y)$ the streamline path $y(x)$ can be found using the ODE: $\frac{d y}{d x}=\frac{v}{u}$, with the seed positions $x_{0}$ and $y_{0}$ for the integration constants. 
In the case of light propagation in an optical fiber, we can solve for the modes of a given fiber geometry using finite element simulations, Comsol v5.1, and examine power flow using the time averaged Poynting vector, the cross product of the electric field and the magnetic field, $\mathbf{E}$ and $\mathbf{H}, \mathbf{S}=\mathbf{E} \times \mathbf{H}$. The longitudinal Poynting vector, $S_{z}(x, y)$, gives the transverse spatial distribution of power as it propagates along the fiber length, producing the familiar mode field distribution, for example compare Fig. 1b with the experimental modefield image in Fig. 1a [2]. The transverse Poynting vectors, $S_{x}(x, y)$ and $S_{y}(x, y)$, represent the flow of power in the transverse plane, we use these vector fields to draw our streamlines. For validation, the radial component of the Poynting vector was integrated along a closed path on the external boundary of the geometry, red curve in Fig. 1c, to give the total power lost. This was within $0.1 \%$ of the confinement loss found from the imaginary part of the mode's effective index. To draw the stream- lines we chose 41 uniformly spaced seed positions near the core to examine one sixth of the geometry. As the lines propagate they show the route the power takes as it leaves the core and ultimately the fiber. Changes in spacing between lines shows local power flux, dense lines are associated with high loss, and low density regions are associated with confinement.

Figure 1c shows the flow lines on a simulated ideal structure closely resembling the fabricated fiber of Fig. 1a [2]. As light propagates, a fraction of the input power slowly flows out of the core following a spiral trajectory. As can be seen, the course of these streamlines changes dramatically when they start to interact with the cladding. Upon reaching the interface with the outer capillary, the flow direction changes and we can see patterns in the flow related to the local cladding structure. Two useful insights emerge from this analysis. The first is that the majority of the leakage (densest streamlines) occurs through the center of the nested capillary; the second is that the flow lines seem to be repelled by and travel around the gap between two large neighboring capillaries. This was initially a surprise to us, as no interface normal to the radial direction and able to repel light exists in that area. Besides, simulations indicate that reducing the size of the gap lowers the loss; consequently, we would have expected these regions of the cross-section to suffer from higher leakage. In contrast, the additional nested element is known to make a dramatic reduction in loss, so why is it associated with high local leakage? A simple physical explanation comes by considering the modes supported in different parts of the cladding; while modes with an effective index not too dissimilar to that of the fundamental core mode exist inside the cavities within the cladding capillaries which favor modal 'coupling' (or light leakage), no such phase matched modes exist in the slender gaps between capillaries.

\section{Designing a new geometry}

Having identified that the gaps between the primary capillaries is efficient at preventing leakage while the nested tube is associated with the highest local leakage, we devised a geometry that replaces high leakage elements with low leakage ones, whilst also being feasible to manufacture.
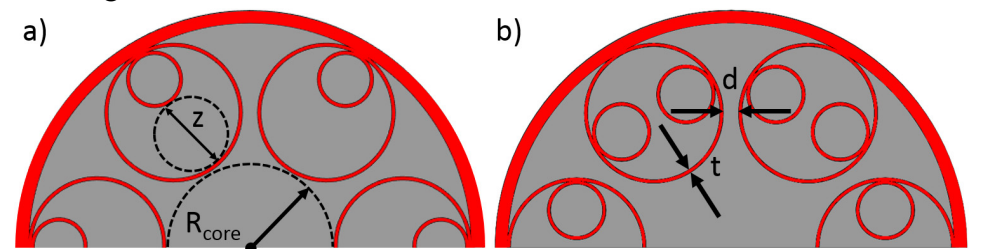

Figure 2. Half diagrams of a) 6 cell NANF geometry, b) the equivalent 6 cell ALIF geometry, hollow regions are shaded grey and glass is red. Both have the same core size, $\mathrm{R}_{\text {core }}=15 \mathrm{um}$, thickness, $t$, and gap, $d$, between adjacent tubes. The size of the first cladding cavity, $\mathrm{z}$, is identified.

We call the new geometry Antiresonant Leakage Inhibited Fiber, ALIF. The single nested element has been replaced by a pair of smaller tubes that are bonded to the inside of the primary element as shown in Fig. 2b. This configuration places a radial gap, known from the discussion above to be a positive confinement feature, into the region which previously had high leakage. Fabrication of this fiber is only slightly more complex than that of a NANF. Six additional inner tubes are needed, but the draw will require the same pressurization strategy which recent fabrications have shown to be well manageable. In contrast, a structure with two capillaries nested inside each other [1] would require an additional pressure region, with a very substantial fabrication complexity overhead.

Figure 3 compares the NANF with a 6 cell and a 5 cell ALIF. All fibers have an identical core size, $R_{\text {core }}=15 \mu \mathrm{m}$, tube thickness, $\mathrm{t}=550 \mathrm{~nm}$, and gap size, $\mathrm{d}=1.5 \mu \mathrm{m}$. The minimum leakage loss of the 6 and 5 tube ALIFs is $\sim 15 \times 10^{-3}$ and $\sim 3 \times 10^{-3} \mathrm{~dB} / \mathrm{km}, \sim 90 \mathrm{x}$ and $\sim 450 \mathrm{x}$ lower than the NANF, respectively. The price to pay for such a remarkable reduction is a narrowing of the useable bandwidth. Resonances associated with the nodes formed where the nested capillaries join the primary capillaries start to appear on the long wavelength side of the transmission window, but they still leave an appreciable $100-200 \mathrm{~nm}$ smooth and resonance-free region.

Reducing from 6 cell to 5 cell enlarges the cladding and moves the jacket glass further away, and in doing so it lowers the loss. Whilst this strategy offers no benefit in the NANF geometry as the enlarged cavities in the cladding starts to support modes that phase match with the core mode, the extra elements in the ALIF cladding geometry reduce the size of the cladding cavities, therefore switching to 5 cells produces a noticeable benefit. 
The power flow lines for the ALIF are presented in Fig. $3 \mathrm{~b}$ using the same parameters as for Fig. $1 \mathrm{~b}$, The low loss of this geometry causes the pitch of the spiraling streamlines to be so tight that they are seen as a solid black shape. The leaking power is still observed to peak near the outer edge of the primary capillary as was seen in the NANF. However, as indicated by the red curve, the leaked power is much lower than for the previous NANF case.
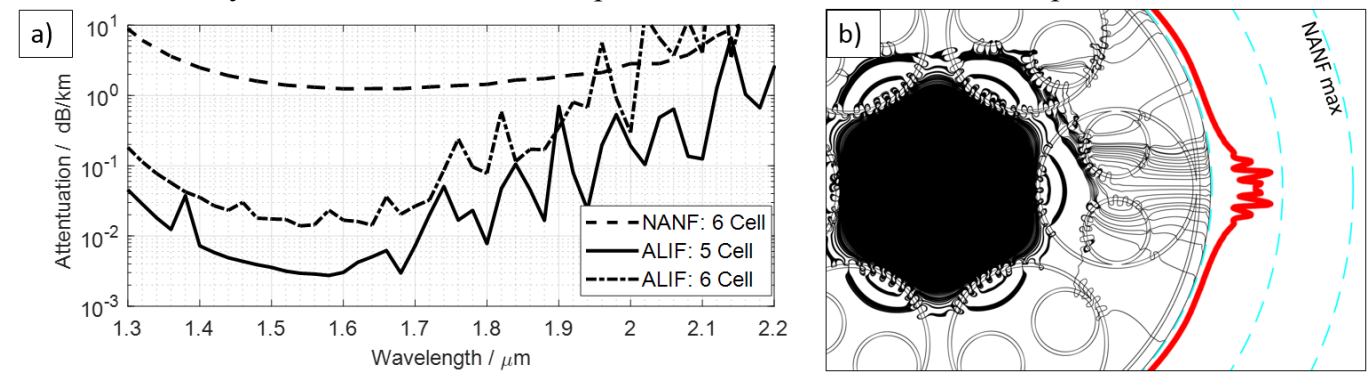

Figure 3. a) The NANF, Fig. 1b, compared with the new ALIF design for 5 and 6 cell, all fibers have core size, $\mathrm{R}_{\text {core }}=15 \mu \mathrm{m}$, thickness, $\mathrm{t}=550 \mathrm{~nm}$, and gap size, $\mathrm{d}=1.5 \mu \mathrm{m}, \mathrm{b}$ ) the streamlines for the 6 cell ALIF with same parameters as Fig. $1 \mathrm{~b}$. The red line shows local power leakage on the same scale as Fig.1b, the blue grid lines have the same magnitude on both figures to allow comparison.

Using the simplified scattering model in [1] we predict that scattering loss for a fiber of this core diameter should be around the loss of SSMF, 0.1-0.15 dB/ km. The exceptionally low confinement loss of the ALIF would then become a negligible contribution, which gives an additional degree of freedom to finely tune its performance. For example, one could make a smaller core, allowing for better compatibility with SMF components, or manipulate the geometry to improve higher order mode suppression, as discussed below.

Effective single mode performance can be achieved when the loss of the higher order modes (HOMs) is 10-100x higher than that of the fundamental core mode. Figure 4 shows the effect that changing the position of the nested elements has on both loss and higher order mode suppression. While keeping the gap between the nested elements constant, we rotated the angular position of their weld point from the primary weld, $\theta$, and calculated the confinement loss of the fundamental mode, and its loss ratio with the LP11 mode. We found that $90^{\circ}$ is the optimal angle for low loss, while $60^{\circ}$ and $120^{\circ}$ are most effective for HOM suppression, reaching a loss ratio $>4500$ (as the ratio $\mathrm{z} / \mathrm{R}_{\text {core }} \sim$ 0.68 [4]) whilst still maintaining extremely low leakage loss. The $90^{\circ}$ structure gives a HOM extinction ratio of $>300$, sufficient for single mode behavior after $\sim 100 \mathrm{~s}$ meters. The position at $60^{\circ}$ generates less resonance than $120^{\circ}$ due to the nodes between nested and primary capillaries becoming further away from the core.

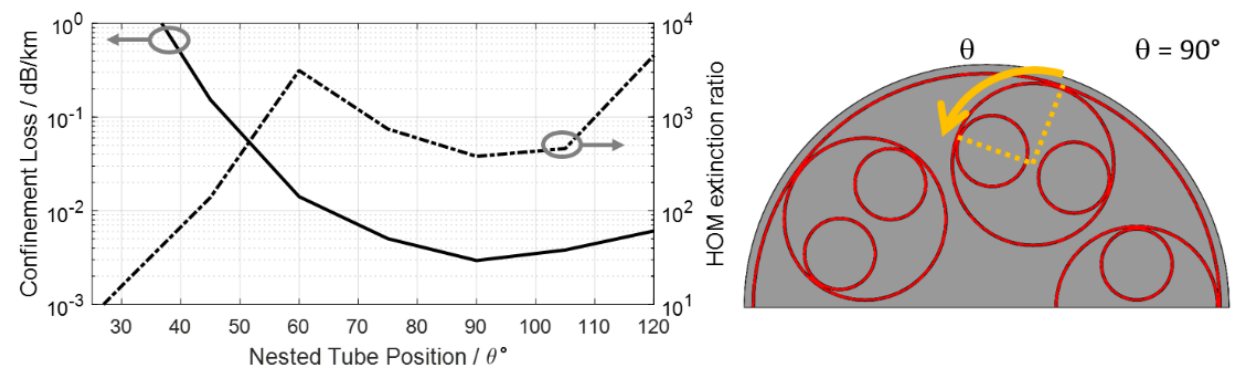

Figure 4. Simulated results of confinement loss and Higher Order Mode extinction for nested tube positions in the 5 cell ALIF, $\lambda=1550 \mathrm{~nm}$

\section{Conclusions}

We have presented a new hollow core antiresonant optical fiber geometry. We used transverse power flow visualization to study the leakage behavior of the recently fabricated NANF geometry. Using this understanding, we devised an improved structure that minimizes leakage loss by exploiting the strong and mitigating the weak geometrical features. The confinement loss of this new geometry is $\sim 450 \mathrm{x}$ better than the equivalent NANF, at a penalty of a reduced but still considerable bandwidth. Having successfully demonstrated fabrication of the NANF design, we are confident that the newly proposed ALIF design can also be fabricated successfully, offering a viable route to truly rival the loss of conventional solid fiber in telecoms applications and beyond.

The authors gratefully acknowledge the support of the Royal Academy of Engineering, ERC (grant agreement no. 682724).

[1] Poletti, Francesco. "Nested antiresonant nodeless hollow core fiber." Optics express 22(20), 23807-23828, (2014).

[2] Bradley T., et al., "Record Low-Loss 1.3dB/km Data Transmitting Antiresonant Hollow Core Fibre", Th3F: Post-deadline papers, European Conference on Optical Communications 2018, Rome, Italy.

[3] Pryamikov, A.D., Alagashev G., "Features of light leakage from the negative curvature hollow core fibers," Opt. Eng. 57(6) 066106, (2018)

[4] Uebel, Patrick, et al. "Broadband robustly single-mode hollow-core PCF by resonant filtering of higher-order modes." Optics letters 41.9, 1961-1964, (2016). 